\title{
Radiofrequency Coil for Dual-nuclei MR Muscle Energetics Investigation Based on Two Capacitively Coupled Periodic Wire Arrays
}

\author{
A. Hurshkainen, M. Dubois, A. Nikulin, C. Vilmen, D. Bendahan, S. Enoch, S. Glybovski and R. Abdeddaim
}

\begin{abstract}
In this paper, we describe a dedicated radiofrequency coil for magnetic resonance imaging and spectroscopy (MRSI) at $4.7 \mathrm{~T}$ optimized for investigation of muscle energetics in a human forearm. The coil operating at the Larmor frequencies of protons ${ }^{1} \mathrm{H}(200.1 \mathrm{MHz})$ and phosphorous ${ }^{31} \mathrm{P}(81$ $\mathrm{MHz}$ ) is based on two cylindrical periodic structures made of thin metal wires with tips interconnected through structural capacities of printed overlapping patches and sliding electric contacts. By independently exciting two orthogonal eigenmodes of the structure, the coil can be tuned and matched at both frequencies without lumped capacitors and cover the target region of interest for the forearm flexor muscles.
\end{abstract}

Index Terms-Radiofrequency coil, wire array, magnetic resonance imaging, magnetic resonance spectroscopy.

\section{INTRODUCTION}

31 -PHOSPHORUS $\left({ }^{31} \mathrm{P}\right)$ Magnetic resonance spectroscopy (MRS) has been largely used over the past decades in order to investigate energy production in muscle tissues [1], [2], [3], [4]. More particularly, a large number of studies has been devoted to the investigation of changes in adenosine triphosphate (ATP), phosphocreatine ( $\mathrm{PCr}$ ) and $\mathrm{pH}$ which are key players in muscle energetics [5], [6], [7]. The low sensitivity of ${ }^{31} \mathrm{P}$-MRS has been largely recognized so that measurements have been mostly performed using small radio frequency (RF) surface coils [8]. These compact magnetic loop antennas attached to investigated regions of interest (ROIs), offer an improved signal-to-noise ratio (SNR) as compared to volume coils surrounding the ROI in many biomedical studies [3], [6], [9], [10]. In the context of muscle energetics studies, investigations have been performed for superficial muscles such as fingers flexor, quadricep femoris and gastrocnemius muscles [6], [9]. For these applications, surface coils have been tailored to cover the ROI. In this context, ${ }^{31} \mathrm{P}$ coils have to be

This work was supported by the European Union's Horizon 2020 research and innovation program under grant agreement No 736937. This research was supported by the Ministry of Education and Science of the Russian Federation (Zadanie No. 3.2465.2017/4.6). This work received funding from the Carnot Star institute. This work is supported by the President of the Russian Federation (MK-3620.2019.8).

Anna Hurshkainen, Anton Nikulin and Stanislav Glybovski were with Department of Physics and Engineering, ITMO University, Saint-Petersburg 197101, Russia (e-mail: s.glybovski@metalab.ifmo.ru).

Marc Dubois, Cristophe Vilmen and David Bendahan was with Aix Marseille Univ, CNRS, Center for Magnetic Resonance in Biology and Medicine, Marseille, France (e-mail: marc.dubois@ fresnel.fr).

Marc Dubois, Redha Abdeddaim and Stefan Enoch were with Aix Marseille Univ, CNRS, Centrale Marseille, Institut Fresnel, Marseille, France (e-mail: abdeddaim@fresnel.fr). combined to proton coils for shimming and/or localization procedures. Dual-nuclei MR studies are also of interest given that metabolites can be detected at both frequencies e.g. ${ }^{1} \mathrm{H}$ and ${ }^{31} \mathrm{P}$. In such case, RF-coils are typically dual-tuned transceiver antennas independently operating at two Larmor frequencies of the given nuclei. Different designs for dual-tuned surface coils have been proposed so far. A single loop operating at two frequencies with independent tuning and matching circuits has been designed [11]. Alternatively, two separate loops could be combined as long as they are decoupled [12], containing a capacitive network for dual-band tuning and matching to 50 $\Omega$. A limitation of conventional loop coils is the depth of the ROI, which is inversely proportional to the loop area, thus coil sensitivity gets maximal at the coil center rapidly decreasing with distance away from this point [8]. However, in the muscle energetics MR studies on a human arm, it is important to localize the ROI nearby the area of the flexor muscles as described in Fig. 1(a). To be exact, the ROI for ${ }^{1} \mathrm{H}$ imaging should cover roughly a half of an arm cross-section to be able to perform single-voxel spectroscopy and anatomical imaging with the same coil. In contrast, at ${ }^{31} \mathrm{P}$ the ROI should coincide with the investigated muscles' cross-section. To remove this limitation, it has been recently proposed to employ hybridized eigenmodes [13], [14] excited in periodic arrays of thin TEM resonators [15], resonant wires or strips [16], [17] and in the open 'bird-cage' structure [18].

In the present work, we report on the design, numerical simulation and experimental demonstration of a genuine dualtuned coil based on two capacitively connected periodic wire arrays. The coil provides the unique combination of a distributed structural capacity together with the advantage of independent geometrical tuning at two Larmor frequencies 81.0 and $200.1 \mathrm{MHz}\left({ }^{1} \mathrm{H} /{ }^{31} \mathrm{P}\right.$ nuclei at $\left.4.7 \mathrm{~T}\right)$ covering the target ROIs for muscle energetics studies.

\section{RF-COIL DESIGN AND CHARACTERIZATION}

The aim of the proposed coil is to fit to the ROI corresponding to the location of flexor muscles in a human arm as shown in Fig. 1(a). The surface area to be covered is around $60 \mathrm{~mm}$ along its bottom semi-circumference and $100 \mathrm{~mm}$ along the arm axis $z$. The arm is placed in the scanner along $B_{0}$ vector and together with the coil must fit to the space available in a narrow preclinical bore of a $4.7 \mathrm{~T}$ MR system (30 $\mathrm{cm}$ diameter). To meet the requirements, the proposed coil operates combining the principles of the surface wire 

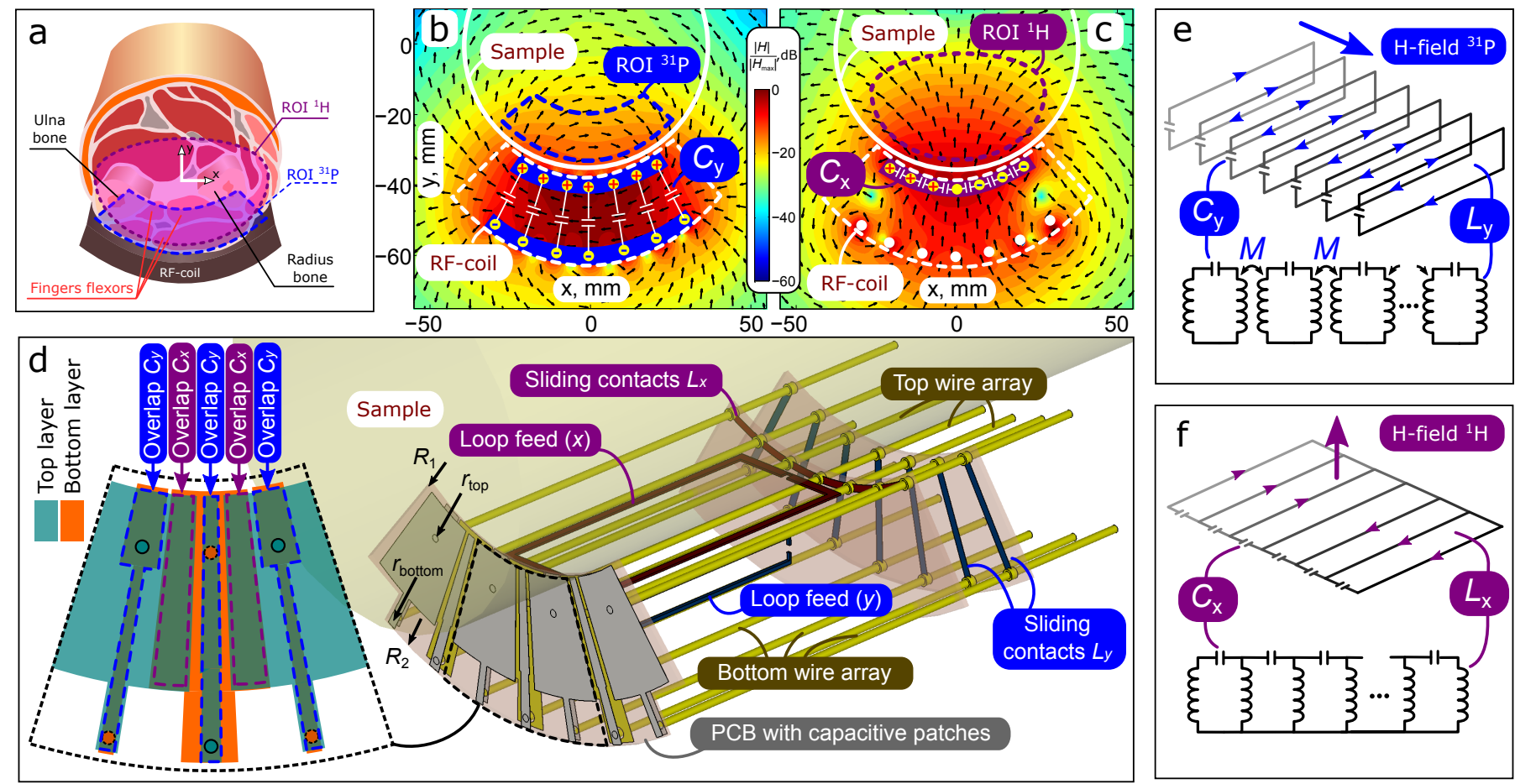

Fig. 1. Application and practical realization of proposed dual-nuclei coil: (a) target regions of interest at ${ }^{31} \mathrm{P}(81 \mathrm{MHz})$ and ${ }^{1} \mathrm{H}(200.1 \mathrm{MHz})$ covering flexor muscles of a human arm; capacitive interconnection of wire ends and corresponding orthogonal-polarized eigenmodes of dual-stage wire array: (b) volume mode for ${ }^{31} \mathrm{P}$, (c) surface mode at ${ }^{1} \mathrm{H}$; (d) practical realization of the proposed coil (inset on the left demonstrates the layout of PCB with capacitive patches for interconnection of wires); (e) wire currents of volume mode and its equivalent circuit; (f) wire currents of surface mode and its equivalent circuit.

resonator, i.e. a single periodic array of resonant coupled wires [16], [19] and the volume wire resonator composed of two separate periodic wire arrays with connected ends in the same structure [20]. In such resonators a number of hybridized eigenmodes can be excited [13]. These eigenmodes differ by their near-field patterns (in particular, by the field confinement nearby the wires) and resonant frequencies. In the proposed coil, two arrays of $N=7$ wires oriented in $z$-direction of the length $150 \mathrm{~mm}$ each, as shown in Fig. 1(d), the top and the bottom ones, were both placed on coaxial cylindrical surfaces with a constant angular periodicity $\alpha=10^{\circ}$ to repeat the shape of a human arm. To make the structure resonant at 81 and $200.1 \mathrm{MHz}$ the tips of all wires on one side of the structure were connected by structural capacities [21]. On the other side of the structure the wires were connected by sliding short-circuiting contacts as shown in Fig. 1(d). Corresponding wires from the top and bottom arrays are connected vertically through structural capacities $C_{y}$, as schematically shown in Fig. 1(b) and also short-circuiting sliding contacts. Due to these connections at the resonant frequency of the fundamental mode the two arrays were excited out of phase confining the magnetic field in between. However, the magnetic field of this mode is also generated outside of the space between the arrays and penetrates into a sample at the distance that depends on the periodicity of the wires. In this regime the resonator can be considered as an array of 7 inductively coupled loops each loaded to a capacity $C_{y}$. This effect was used to localize the ROI at $81 \mathrm{MHz}$. The magnetic field $\mathbf{H}$ of this volume mode in the sample has a horizontal polarization and its normalized magnitude pattern in the axial plane is shown in Fig. 1(b). The wire currents directions and the equivalent circuit corresponding to the volume mode are depicted in Fig. 1(e). The equivalent circuit of the volume mode is represented by 7 inductively coupled LC-circuits based on the structural fixed capacitance $C_{y}$ and inductance $L_{y}$ of an individual rectangular loop of current consisting of one wire from the top and one from the bottom layer as well as the short-circuit slider. In the same wire structure another mode with a vertical polarization can be excited with the magnetic field pattern depicted in Fig. 1(c). To enable this surface mode at 200.1 $\mathrm{MHz}$ each pair of neighbouring wire ends in the top array was connected horizontally by capacities $C_{x}$ as shown in Fig. 1(c). Current directions on the top layer of wires and the corresponding equivalent circuit of the surface mode are shown in Fig. 1(f). The equivalent circuit in this case is represented by a chain of 6 high-pass LC circuits, where in addition to the fixed structural capacitance $C_{x}$ in each unit cell there is a wire inductance depending on the position of the sliding short-circuit. Both the capacities $C_{x}$ and $C_{y}$ were fixed and realized using overlapped patches placed on the opposite sides of a printed-circuit board (PCB) with a 1.5-mm-thick Rogers RT/Duroid 6010 substrate $\left(\varepsilon_{\mathrm{r}}=10.7\right.$ and $\left.\tan \delta=0.0023\right)$. The overlap regions on the PCB are responsible for both types of capacity are shown in the inset on the left of Fig. 1(d).

Every RF-coil for biomedical research requires the possibility to adjust tuning and matching to compensate in the experiment for the sample properties' variation. In the proposed coil, fine frequency tuning of both modes is achieved by moving the horizontal and vertical sliding contacts shown in Fig. 
1(d) to mechanically adjust the structural inductances $L_{x}$ and $L_{y}$ in the equivalent circuits of Fig. 1(e,f), correspondingly. The position of each slider changes the distributed inductance and controls the mode frequencies ( $L_{x}$ individually for the surface mode, while $L_{y}$ individually for the volume mode) with the tunability of $1 \mathrm{MHz} / \mathrm{mm}$. Thanks to the wide range of distributed inductance values the coil is appropriate for biomedical applications allowing frequency tuning for the large range of sample volumes. Moreover, independent tuning for the selected modes is possible because $L_{x}$ short-circuits only top-array wires, while $L_{y}$ pair the corresponding wires which belong to different arrays. The resonant frequencies of the volume and surface fundamental modes can be estimated from the equivalent circuits shown in Fig. 1(e,f). The circuit estimations differ from numerical simulations by $20-30 \%$ due to the presence of the sample and parasitic reactances in the structure. Finally the exact positions of both sliding contacts were chosen by numerical optimization.

Both modes are excited by two mutually orthogonal feeding loops which areas affect the input impedance of two feeding points. The vertical loop (Y) excites the volumetric mode, while the horizontal loop (X) couples to the surface mode. The loops were made expandable in $z$-direction thanks to telescopic sliding tubes to adjust the coil matching to $50 \Omega$ at both frequencies. By numerical optimization in CST Studio Suite, the following structural parameters of the PCB and sliders were chosen: the radii of the top and bottom wire arrays $r_{\text {top }}=60 \mathrm{~mm}, r_{\text {bottom }}=90 \mathrm{~mm}$ and the PCB internal and external radii $R_{1}=50 \mathrm{~mm}, R_{2}=93 \mathrm{~mm}$. The distances from the sliding contacts to the PCB plane were $L_{x}=98$ $\mathrm{mm}, L_{y}=109 \mathrm{~mm}$. All wires were made of brass and had the diameter of a circular cross-section of $2 \mathrm{~mm}$. The coil was considered in the simulation inside a cylindrical MR-system bore of the diameter $30 \mathrm{~cm}$ with a homogeneous cylindrical phantom $\left(\varepsilon_{p}=34\right.$ and $\left.\sigma_{p}=0.4 \mathrm{~S} / \mathrm{m}\right)$ having the the radius $R_{p}=50 \mathrm{~mm}$ and length $L_{p}=250 \mathrm{~mm}$ representing a human arm. For the above numerically optimized geometric parameters of the PCB and wires of the coil one can estimate the inductance of a loop between one top and one bottom wire as $L_{y}=188 \mathrm{nH}$, and the structural overlap capacitance $C_{y}=7.8 \mathrm{pF}$ predicting for $M=0.95 L_{y}$ the fundamental volume mode resonance at $95 \mathrm{MHz}$. By taking the structural patch overlap capacity $C_{x}=10.1 \mathrm{pF}$ and the inductance of the top-layer wire of $L_{x}=48 \mathrm{nH}$, one estimates the resonant frequency of the surface mode equal to $260 \mathrm{MHz}$. However, the precise resonant frequencies calculated in CST equal 80.1 and $200 \mathrm{MHz}$. Indeed, the simulated S-parameters represented in Fig. 2 show that the coil at both $50-\Omega$ ports connected to the loops $(\mathrm{X})$ and $(\mathrm{Y})$ with the chosen parameters is simultaneously tuned and matched at two Larmor frequencies. The calculated coefficient $S_{12}$ of parasitic coupling between the two ports was negligible due to orthogonality of the excited eigenmodes and therefore it is not presented in Fig. 2. As can be seen from Fig. 2, the relative bandwidth of matching for ${ }^{1} \mathrm{H}$ nucleus is much higher than for ${ }^{31} \mathrm{P}$ one.

The calculated magnitude patterns of $B_{1}^{+}$field, i.e. a righthanded circularly polarized component of magnetic RF-field, normalized by $1 \mathrm{~W}$ of accepted power, created by the coil

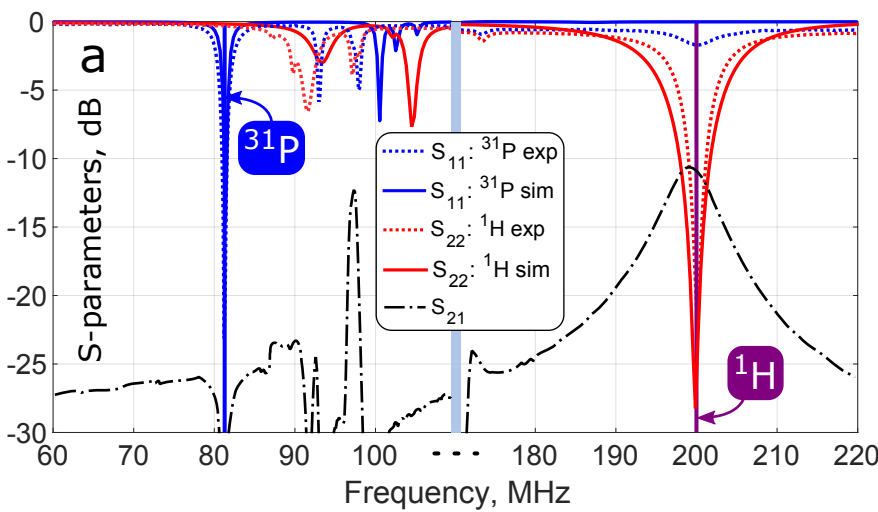

Fig. 2. Simulated and measured $S_{11}$ (reflection coefficient at ${ }^{31} \mathrm{P}$ port) and $S_{22}$ (reflection coefficient at ${ }^{1} \mathrm{H}$ port) and measured $S_{21}$ (transmission coefficient between two ports) of the proposed coil in two frequency ranges: from 60 to 120 and from 170 to $220 \mathrm{MHz}$.

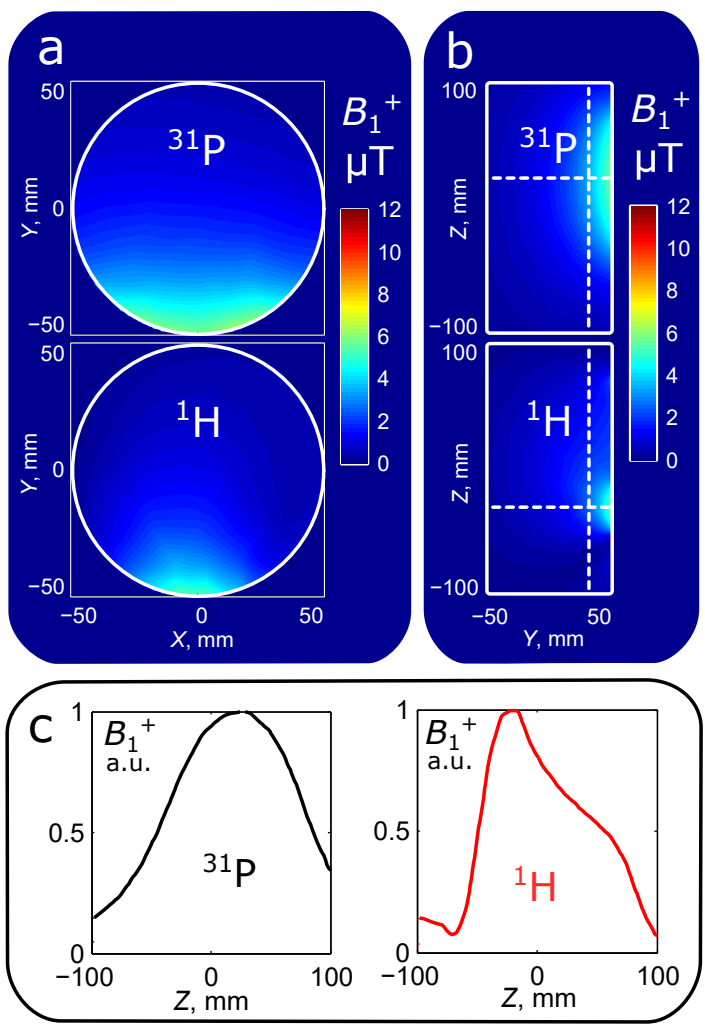

Fig. 3. Simulation results of the proposed coil at two Larmor frequencies: $B_{1}+$ patterns for $1 \mathrm{~W}$ of accepted power in transverse (a) and sagittal (b) planes; $B_{1}+$ profiles for $1 \mathrm{~W}$ of accepted power in $z$-direction at the depth $25 \mathrm{~mm}$ in the phantom $(y=-25 \mathrm{~mm})$.

at the Larmor frequencies, are shown in Fig. 3(a,b) in the transverse $(X Y)$ and sagittal $(Y Z)$ planes of the phantom correspondingly. Normalized profiles of $B_{1}^{+}$field along $z$ direction are given in Fig. 3(c) for the depth $25 \mathrm{~mm}$ in the phantom. The corresponding coordinate line is shown by vertical dashed lines in Fig. 3(b). Due to the calculated transmit field patterns and profiles one can conclude that the proposed coil has the required coverage area with the length of $100-120 \mathrm{~mm}$ in $z$-direction and around $60 \mathrm{~mm}$ in the arm circumference direction. The coverage area is defined as 


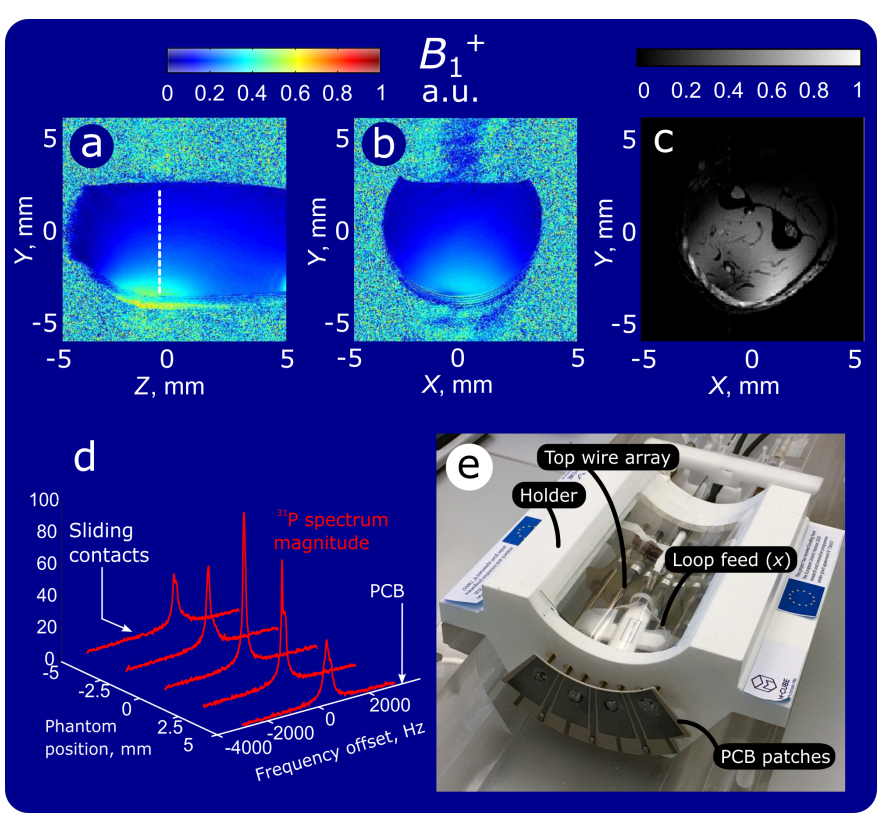

Fig. 4. Experiment: $B_{1}^{+}$field maps for ${ }^{1} \mathrm{H}$ in sagittal (a) and transverse (b) planes; in-vivo images of a human forearm in the transverse plane (c); ${ }^{31} \mathrm{P}$ spectra of a test-tube phantom depending on its position in $z$-direction (d); (e) prototype of the proposed coil.

the area where $B_{1}^{+}$magnitude holds higher than $50 \%$ of the maximum of the whole pattern. In other words, the proposed coil creates relatively homogeneous field in the half of the phantom's axial cross-section, while producing the magnetic field in the ROI with the required shape closer to the bottom surface at ${ }^{31} \mathrm{P}$.

To experimentally characterize the proposed coil, its prototype has been manufactured and the results of the measurements are presented in Fig. 4. The prototype shown in Fig. 4(e) was built of 14 brass wires with the capacitive PCB soldered to their ends, two sliding contacts for precise dual-band tuning and two feeding loops soldered to two coaxial cables for connecting to two frequency channels of the MR-systems' transceiver. All the parts were mounted on a 3D-printed holder with a shape conformal to a human arm. On-bench measurements of S-parameters were done for each channel with a vector network analyzer (VNA MS2036C, Anritsu). $\left|S_{11}\right|$ and $\left|S_{22}\right|$ parameters represent the reflection coefficients from the vertical and horizontal feed loop ports, respectively, while $\left|S_{21}\right|$ characterizes the isolation of the two ports. In the measurements both sliding contacts were adjusted for proper dual-band tuning. The coil was loaded by a $500 \mathrm{~mL}$ glass jar containing distilled water with a 0.1 mol. $\mathrm{L}^{-1}$ concentration of phosphorus nuclei from dissolved poly-phosphoric acid. Comparison of the measured S-parameters to the calculated ones shown in Fig. 2 is very good and confirms the possibility of simultaneous tuning to 81 and $200.1 \mathrm{MHz}$ and matching to $50 \Omega$ at both frequencies without using lumped capacitors. Moreover, the measured $\left|S_{21}\right|$ holds below $-11 \mathrm{~dB}$ at any frequency, showing that the two ports are sufficiently isolated due to the orthogonality of the excited modes.

To map the RF-field patterns created by the proposed coil and test its imaging and spectroscopy capabilities, a set of
MR acquisitions were performed at $4.7 \mathrm{~T}$ on $47 / 30$ Biospec Avance MR system (Bruker, Karlsruhe, Germany). Fast Low Angle shot (FLASH) gradient echo sequences were performed in sagittal and axial orientations of the phantom with $\mathrm{T}_{R} / \mathrm{T}_{E}$ of $4.3 / 500 \mathrm{~ms}, 5 \mathrm{~mm}$ slice thickness, $12 \times 12 \mathrm{~cm}^{2}$ FOV with 0.625 $\mathrm{mm}^{2}$ pixels. This sequence was repeated with nine different nominal flip angles ranging from $1.5^{\circ}$ to $30^{\circ}$. These series of images allowed us to retrieve the relative $B_{1}^{+}$amplitude, i.e. the transmit efficiency of the coil (proportional to the ratio of $\mathrm{B}_{1}^{+}$to the square root of input power in the impedancematched regime). This method was already described [22] and successfully implemented for $\mathrm{B}_{1}^{+}$maps retrieval [20]. The measured relative $B_{1}^{+}$patterns at ${ }^{1} \mathrm{H}$ frequency are depicted in Fig. 4 for the axial (a) and sagittal (b) slices crossing the maximum. The measured field patterns are in good agreement with the numerical results. In order to illustrate how the increased FOV depth affect an anatomical hydrogen MR image of a human arm, and in-vivo scan using a FLASH sequence with $\mathrm{T}_{R} / \mathrm{T}_{E}$ of $500 / 10 \mathrm{~ms}$, a voxel size $0.54 \times 0.54 \mathrm{~mm}^{2}$ and the slice thickness of $5 \mathrm{~mm}$ and a FOV of $103.8 \times 103.8 \mathrm{~mm}^{2}$ was performed. As expected, the proposed coil creates a FOV covering almost half of the arm transverse cross-section which is illustrated in Fig. 4(c). To characterize the proposed coil at ${ }^{31} \mathrm{P}$, single-voxel spectroscopy has been made using a small 5 $\mathrm{mL}$ test tube filled with the same liquid as mentioned above. A large coverage area provided by the coil can be clearly seen in Fig. 4(d) where obtained single-voxel spectra of a small test tube are presented depending on the position of the latter in $z$-directions with respect to the coil. Indeed, for the proposed coil the spectrum holds higher than $50 \%$ of the maximum value within a range of $100 \mathrm{~mm}$ in $z$-direction.

\section{CONCLUSION}

In conclusion, a new dual-frequency RF-coil operating at 81.0 and $200.1 \mathrm{MHz}$ for muscle energetics MR investigation has been designed and the corresponding performance has been characterized. Due to the orthogonality of its excited surface and volumetric modes, independent tuning and matching of its ${ }^{31} \mathrm{P}$ and ${ }^{1} \mathrm{H}$ channels was shown. This possibility along with a structural capacity of printed overlap patches is a unique combination among the known dual-tuned surface coils for MRI/MRS. The proposed coil precisely covers the region of flexor muscles in a human arm at the Larmor frequency of ${ }^{31} \mathrm{P}$ due to a relatively confined RF field of the volume mode. This helps in spectroscopy methods to selectively receive RF signals from those investigated muscles. At the same time it is homogeneously enough covers the whole arm crosssection at the Larmor frequency of protons, thus, providing the possibility to obtain reference anatomical maps of a subject.

\section{REFERENCES}

[1] A. Boss, L. Heskamp, V. Breukels, L. J. Bains, M. J. van Uden, and A. Heerschap, "Oxidative capacity varies along the length of healthy human tibialis anterior," The Journal of Physiology, vol. 596, no. 8, pp. 1467-1483, 2018 .

[2] G. Kemp, R. Ahmad, K. Nicolay, and J. Prompers, "Quantification of skeletal muscle mitochondrial function by 31P magnetic resonance spectroscopy techniques: a quantitative review," Acta Physiologica, vol. 213 , no. 1 , pp. 107-144, 2015. 
[3] G. Layec, L. J. Haseler, J. Hoff, C. R. Hart, X. Liu, Y. Le Fur, E.-K. Jeong, and R. S. Richardson, "Short-term training alters the control of mitochondrial respiration rate before maximal oxidative ATP synthesis," Acta Physiologica, vol. 208, no. 4, pp. 376-386, 2013.

[4] K. Yashiro, A. Tonson, É. Pecchi, C. Vilmen, Y. Le Fur, M. Bernard, D. Bendahan, and B. Giannesini, "Capsiate supplementation reduces oxidative cost of contraction in exercising mouse skeletal muscle in vivo," PloS One, vol. 10, no. 6, p. e0128016, 2015.

[5] G. Layec, A. Bringard, Y. Le Fur, J.-P. Micallef, C. Vilmen, S. Perrey, P. J. Cozzone, and D. Bendahan, "Opposite effects of hyperoxia on mitochondrial and contractile efficiency in human quadriceps muscles," American Journal of Physiology-Heart and Circulatory Physiology, 2015.

[6] G. Layec, E. Malucelli, Y. Le Fur, D. Manners, K. Yashiro, C. Testa, P. J. Cozzone, S. Iotti, and D. Bendahan, "Effects of exercise-induced intracellular acidosis on the phosphocreatine recovery kinetics: a 31P mrs study in three muscle groups in humans," NMR in Biomedicine, vol. 26, no. 11, pp. 1403-1411, 2013.

[7] D. W. Russ and J. A. Kent-Braun, "Is skeletal muscle oxidative capacity decreased in old age?," Sports Medicine, vol. 34, no. 4, pp. 221-229, 2004.

[8] J. J. Ackerman, T. H. Grove, G. G. Wong, D. G. Gadian, and G. K. Radda, "Mapping of metabolites in whole animals by 31P NMR using surface coils," Nature, vol. 283, no. 5743, p. 167, 1980.

[9] D. Taylor, P. Bore, P. Styles, D. Gadian, and G. Radda, "Bioenergetics of intact human muscle. a 31P nuclear magnetic resonance study.," Molecular Biology \& Medicine, vol. 1, no. 1, pp. 77-94, 1983.

[10] P. Barnes, G. J. Kemp, D. J. Taylor, and G. K. Radda, "Skeletal muscle metabolism in myotonic dystrophy a 31P magnetic resonance spectroscopy study.," Brain: a Journal of Neurology, vol. 120, no. 10, pp. 1699-1711, 1997.

[11] F. D. Doty, G. Entzminger, J. Kulkarni, K. Pamarthy, and J. P. Staab, "Radio frequency coil technology for small-animal MRI," NMR in Biomedicine, vol. 20, no. 3, pp. 304-325, 2007.

[12] G. Adriany and R. Gruetter, "A half-volume coil for efficient proton decoupling in humans at 4 Tesla," Journal of Magnetic Resonance, vol. 125 , no. 1 , pp. $178-184,1997$.

[13] C. Jouvaud, R. Abdeddaim, B. Larrat, and J. De Rosny, "Volume coil based on hybridized resonators for magnetic resonance imaging," Applied Physics Letters, vol. 108, no. 2, p. 023503, 2016.

[14] M. Dubois, L. Leroi, Z. Raolison, R. Abdeddaim, T. Antonakakis, J. De Rosny, A. Vignaud, P. Sabouroux, E. Georget, B. Larrat, et al., "Kerker effect in ultrahigh-field magnetic resonance imaging," Physical Review X, vol. 8, no. 3, p. 031083, 2018.

[15] A. S. Peshkovsky, R. P. Kennan, M. E. Fabry, and N. I. Avdievich, "Open half-volume quadrature transverse electromagnetic coil for highfield magnetic resonance imaging," Magnetic Resonance in Medicine, vol. 53, no. 4, pp. 937-943, 2005.

[16] A. P. Slobozhanyuk, A. N. Poddubny, A. J. Raaijmakers, C. A. van Den Berg, A. V. Kozachenko, I. A. Dubrovina, I. V. Melchakova, Y. S. Kivshar, and P. A. Belov, "Enhancement of magnetic resonance imaging with metasurfaces," Advanced Materials, vol. 28, no. 9, pp. 1832-1838, 2016.

[17] A. Hurshkainen, A. Nikulin, E. Georget, B. Larrat, D. Berrahou, A. L. Neves, P. Sabouroux, S. Enoch, I. Melchakova, P. Belov, et al., "A novel metamaterial-inspired RF-coil for preclinical dual-nuclei MRI," Scientific Reports, vol. 8, no. 1, p. 9190, 2018.

[18] A. Yahya, N. De Zanche, and P. S. Allen, "A dual-tuned transceive resonator for 13C1H MRS: two open coils in one," NMR in Biomedicine, vol. 26, no. 5, pp. 533-541, 2013.

[19] E. A. Brui, A. V. Shchelokova, M. Zubkov, I. V. Melchakova S. B. Glybovski, and A. P. Slobozhanyuk, "Adjustable subwavelength metasurface-inspired resonator for magnetic resonance imaging," Physica Status Solidi (a), vol. 215, no. 5, p. 1700788, 2018.

[20] A. V. Shchelokova, C. A. van den Berg, D. A. Dobrykh, S. B. Glybovski, M. A. Zubkov, E. A. Brui, D. S. Dmitriev, A. V. Kozachenko, A. Y. Efimtcev, A. V. Sokolov, et al., "Volumetric wireless coil based on periodically coupled split-loop resonators for clinical wrist imaging," Magnetic Resonance in Medicine, vol. 80, no. 4, pp. 1726-1737, 2018

[21] S. B. Glybovski, A. V. Shchelokova, A. V. Kozachenko, A. P. Slobozhanyuk, I. V. Melchakova, P. A. Belov, A. V. Sokolov, A. Y. Efimtsev, and V. A. Fokin, "Capacitively-loaded metasurfaces and their application in magnetic resonance imaging," in 2015 Radio and Antenna Days of the Indian Ocean (RADIO), pp. 1-2, Sep. 2015.

[22] L. W. Bartels, C. J. Bakker, and M. A. Viergever, "Improved lumen visualization in metallic vascular implants by reducing RF artifacts," Magnetic Resonance in Medicine, vol. 47, no. 1, pp. 171-180, 2002. 